\title{
GMR
}

\section{Prognostic significance of microRNA-10b overexpression in breast cancer: a meta- analysis}

\author{
N. Wang ${ }^{1}$, P. Chen ${ }^{1}$, L.P. Huang ${ }^{1}$ and T.Z. Wang ${ }^{2}$ \\ 'Department of Breast and Thyroid Surgery, China-Japan Friendship Hospital, \\ Beijing, China \\ ${ }^{2}$ Hospital of Renmin University of China, Beijing, China \\ Corresponding author: N. Wang \\ E-mail: ningwang72@126.com
}

Genet. Mol. Res. 15 (2): gmr.15027350

Received July 30, 2015

Accepted November 17, 2015

Published April 4, 2016

DOI http://dx.doi.org/10.4238/gmr.15027350

ABSTRACT. Many microRNAs (miRNAs) exhibit altered expression levels in cancers, and they may be considered as valuable prognostic biomarkers for cancers. Here we aimed to summarize the recent advances in miR$10 \mathrm{~b}$ involvement in human breast cancer and analyze the predicting role of miR-10b for survival. We searched, Embase, and Wanfang databases to identify studies on the prognostic role of miR-10b expression in breast cancer. A total of 770 patients from 7 eligible studies were included in the analysis. Pooled risk ratios (RRs) with $95 \%$ confidence interval $(95 \% \mathrm{Cl})$ were calculated to estimate the effect. Our results showed that high miR$10 \mathrm{~b}$ expression in patients with breast cancer was significantly associated with poor disease-free survival (DFS) $(R R=1.53 ; 95 \% \mathrm{Cl}=1.06-2.21 ; \mathrm{P}$ $=0.02$ ). However, no significant association between miR-10b and overall survival was found in overall studies. Subgroup analysis indicated that high expression of miR-10b was significantly correlated with DFS in Asia (RR $=2.94 ; 95 \% \mathrm{Cl}=1.71-5.05)$. The present meta-analysis demonstrated that high expression of miR-10b might predict poor survival in patients with 
breast cancer. Larger clinical studies are required to further evaluate the role of miR-10b in clinical practice.

Key words: MicroRNA-10b; Breast cancer; Prognostic factor; Meta-analysis

\section{INTRODUCTION}

Mortality from breast cancer has declined in the western counties over the last 30 years likely as a result of effective early detection due to more widespread screening efforts and advances in adjuvant therapy (Harbeck, 2007). However, breast cancer remains a leading cause of cancer-related mortality in females worldwide. Traditionally, breast cancers have been classified into prognostically meaningful groups based on clinical features and histopathological findings, but it is increasingly evident that cellular and molecular characteristics are of significant importance (Karlsson et al., 2013).

MicroRNAs (miRNAs) are small non-coding RNAs (18-25 nucleotides in length) that act as complex negative regulators of posttranscriptional gene expression by targeting regions of sequence similarity in mRNA 3'-untranslated regions, leading to either degradation or translational inhibition of the mRNA (Foley et al., 2011). Recent studies have shown that miRNAs were involved in various physiological and pathological processes, and an increasing number of evidence prove that the abnormal expression of miRNAs has a direct correlation with the occurrence and development of cancers. The abnormal expression and dysfunction of miRNAs may lead to cellular disorder and finally result in diseases or even cancers.

The miR-10b gene is located in the middle of the HOXD cluster on chromosome $2 q 31$, near HOXD4 (Ma et al., 2007). A combination of mouse and human cells exhibited that miR-10b is highly expressed in metastatic breast cancer cells and positively regulates cell migration and invasion (Ma et al., 2007). Overexpression of miR-10b in otherwise non-metastatic breast tumors initiates robust invasion and metastasis, and the level of miR-10b expression in primary breast carcinomas correlates with clinical progression (Sasayama et al., 2009).

Over the past few years, several studies have evaluated the prognostic value of miR-10b expression in breast cancer with conflicting results. Some concluded that miR-10b expression had no influence on survival (Gee et al., 2008; Radojicic et al., 2011; Parrella et al., 2014), while others reported that miR-10b expression was predictive of decreased survival outcome for breast cancer (Liang et al., 2011; Chang et al., 2014). Insufficient samples and some other factors have resulted in controversial results of different clinical studies. Thus, the present meta-analysis was performed to determine the value of miR10b as a prognostic marker for breast cancer.

\section{MATERIAL AND METHODS}

\section{Literature search strategy}

We comprehensively searched, Embase, and Wanfang databases for relevant articles published until July 1st, 2015. The search strategy included the following key words: miR-10b, microRNA-10b, breast cancer, breast carcinoma, prognostic and prognosis. The citation lists associated with all the studies retrieved in the search were used to identify other potentially relevant publications. Review articles were also scanned to find additional eligible studies. The title and abstract of each study identified in the search were scanned to exclude any clearly irrelevant 
publications. The remaining articles were browsed to determine whether they contained information on the topic of interest.

\section{Criteria for inclusion and exclusion}

The following inclusion criteria were used in order to ensure the high quality of this article: 1) patients with breast cancer diagnosis by pathology; 2) full-length paper with sufficient data on survival and miR-10b expression; 3) miR-10b expression was measured by real time-PCR method. The following studies were excluded: 1) articles about cell lines or animals; 2) review articles without original data; 3 ) studies lacking information on survival.

\section{Data extraction}

Information was carefully extracted from all eligible publications independently by two of the authors according to the inclusion criteria listed above. Disagreement was resolved by a consensus discussion between the two authors. Data tables were composed to extract all relevant data from texts, tables, and figures of each included study, including author, publication year, country of patient's origin, time of collection tumor stage, number of patients, the age of patients, follow-up months, cut-off value of overexpression of miR-10b, and survival analysis. In case, the prognosis was only plotted as Kaplan-Meier curve in some articles, the GetData Graph Digitizer 2.24 software (free software downloaded for http://getdata-graph-digitizer.com) was applied to digitize and extract the data.

\section{Statistical analysis}

Pooled risk ratios $(\mathrm{RR})$ and $95 \%$ confidence interval $(95 \% \mathrm{Cl})$ were used as the effective value to measure the impact of miR-10b expression on survival of breast cancer patients in this meta-analysis. In the individual study, some of them provided RR and $95 \% \mathrm{Cl}$ directly. Heterogeneity was assessed by the chi-squared test. The $\mathrm{I}^{2}$ value was used to evaluate the heterogeneity $\left(\mathrm{I}^{2}=\right.$ $0-50 \%$, no or moderate heterogeneity; $\left.\right|^{2}>50 \%$, significant heterogeneity) (Higgins et al., 2003). The Begg test and the Egger test were performed to identify the possibility of publication bias (Begg and Mazumdar, 1994; Egger et al., 1997). Fixed-effect model was used if there was no significant heterogeneity. Otherwise, the random-effect model was used. Sensitivity analysis was performed to examine the stability of the pooled results. By convention, an observed $R R>1$ implied a poor survival for the group with increased miR-10b expression. All analyses were executed using the STATA 12.0 software (2008; Stata Statistical Software: Version 12.0, StataCorp., College Station, TX, USA).

\section{RESULTS}

\section{Characteristics of the studies}

Using the search criteria (Figure 1), a total of 7 publications met the criteria for this analysis (Gee et al., 2008; Radojicic et al., 2011; Liang et al., 2011; Song et al., 2012; Chang et al., 2014; Markou et al., 2014; Parrella et al., 2014). The total number of patients included was 770 ranging from 60 to 219 patients per study. Among the 7 studies, three studies were performed in Asian populations, and the remaining four studies followed Caucasian patients. All patients in the 
eligible studies were determined by pathological stage. All of the studies reported the prognostic value of miR-10b expression for survival in patients with breast cancer. Of the 7 studies, one directly reported survival data, while the other six studies provided survival curves. Estimation using survival curves were segregated according to either disease-free survival (DFS) or overall survival (OS). ARR on DFS or OS could be extracted for all enrolled studies. The characteristics of the included studies are listed in Table 1.

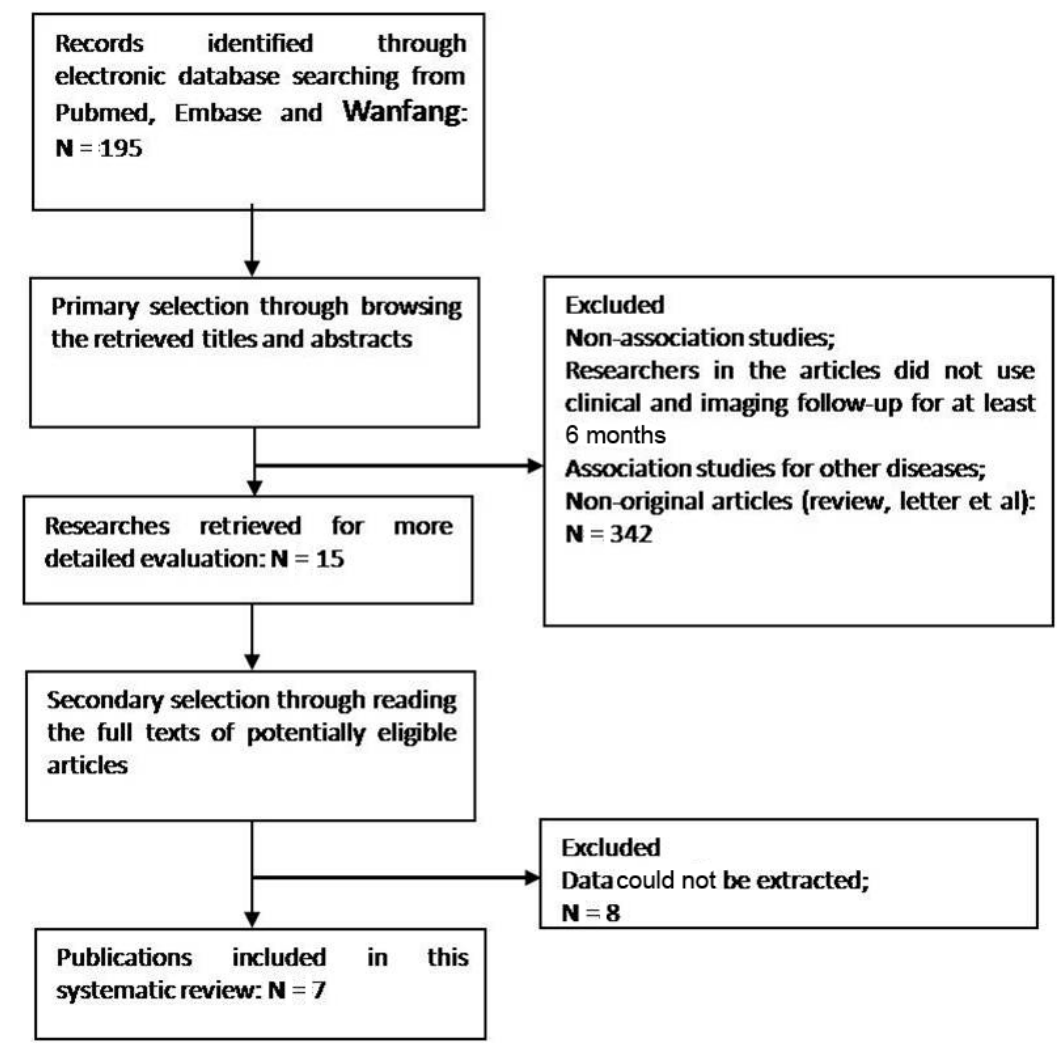

Figure 1. Flow diagram of article selection.

Table 1. Main characteristics and results of the eligible studies.

\begin{tabular}{|c|c|c|c|c|c|c|c|c|c|}
\hline Study & \begin{tabular}{|l|} 
Patient's \\
country
\end{tabular} & Year & $\begin{array}{c}\text { Time of } \\
\text { collection }\end{array}$ & $\begin{array}{c}\text { Pathological } \\
\text { stage }\end{array}$ & $\begin{array}{c}\text { No. of } \\
\text { patients }\end{array}$ & $\begin{array}{l}\text { Age in } \\
\text { years }\end{array}$ & $\begin{array}{c}\text { Follow-up } \\
\text { months }\end{array}$ & Cut-off & $\begin{array}{l}\text { Survival } \\
\text { analysis }\end{array}$ \\
\hline Gee 2008 & UK & 2008 & 1989-1992 & 1 & 219 & ND & 180 & Median & DFS \\
\hline Liang 2011 & China & 2011 & $2004-2008$ & I-IV & 83 & ND & 78 & Mean & DFS \\
\hline Chang 2014 & Taiwan & 2014 & 1994-2013 & I-IV & 108 & $35-102$ & 96 & $\begin{array}{l}\text { ROC } \\
\text { value }\end{array}$ & DFS \\
\hline Radojicic 2011 & Greece & 2011 & ND & ND & 87 & ND & 120 & Mean & OS; DFS \\
\hline Parrella 2014 & Italy & 2014 & $2006-2011$ & I-IV & 101 & $36-82$ & 36 & Median & OS; DFS \\
\hline Song 2012 & China & 2012 & $2002-2005$ & I-III & 60 & $31-92$ & 120 & Mean & OS; DFS \\
\hline Markou 2014 & Greece & 2014 & ND & I-IV & 112 & ND & 149 & Median & OS; DFS \\
\hline
\end{tabular}

ND = not documented. DFS = disease-free survival; OS = overall surviral. 


\section{miR-10b expression and prognosis of breast cancer}

With the use of the methods described above, the OS and/or DFS of 770 patients in the 7 studies were analyzed. The main results of this meta-analysis are shown in Figure 2. A fiveyear DFS rate was extracted from seven studies. The meta-analysis of the seven studies for the prognostic value of miR-10b expression showed that miR-10b overexpression is associated with poor DFS $(\mathrm{RR}=1.53,95 \% \mathrm{Cl}=1.06-2.21, \mathrm{P}=0.02$; Figure $2 \mathrm{~A})$.

However, the meta-analysis of four applicable studies showed that miR-10b overexpression is not significantly associated with $\mathrm{OS}(\mathrm{RR}=1.05,95 \% \mathrm{Cl}=0.67-1.64, \mathrm{P}=0.85$; Figure $2 \mathrm{~B})$.

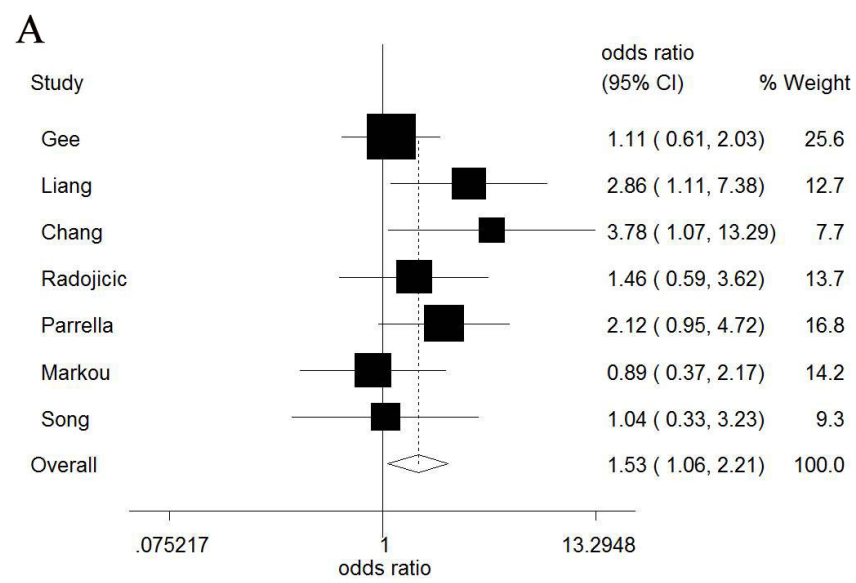

$\mathrm{B}$

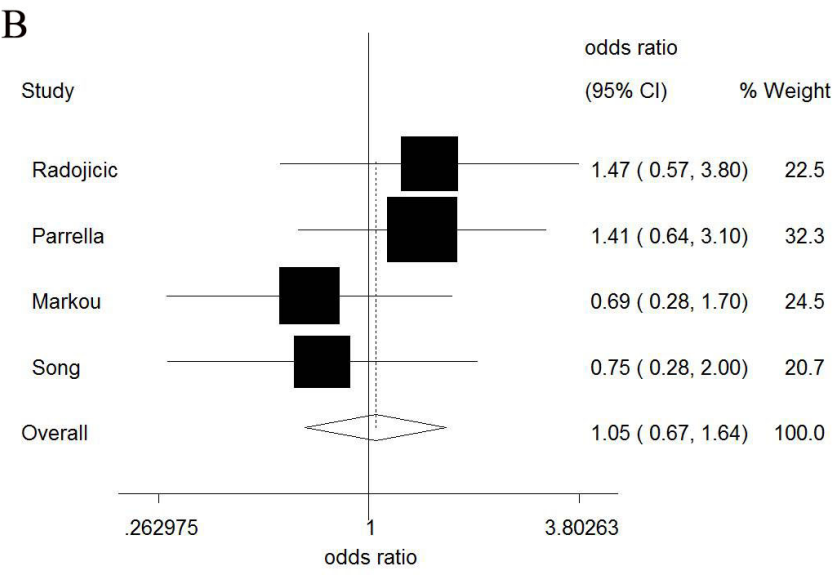

Figure 2. Analysis of miR-10b expression and survival of breast patients. Forest plot of RR for disease-free survival (A) and overall survival (B) among included studies.

Table 2 shows the results of the subgroup meta-analyses. When grouped according to the ethnicity, the combined RRs of Asian studies in DFS were $2.94(95 \% \mathrm{Cl}=1.71-5.05)$, which shows that miR-10b is an indicator of poor DFS prognoses in Asian patients. In addition, when miR-10b expression level was stratified by mean value, high miR-10b expression is associated with poor DFS $(R R=1.75 ; 95 \% \mathrm{Cl}=1.16-2.62)$ in breast cancer patients. 
Table 2. Subgroup analysis of the studies reporting the prognostic value of miRNA-10b expression on DFS of breast cancer.

\begin{tabular}{l|c|c|c|c|c|c|c}
\hline Variables & $\begin{array}{c}\text { No. of } \\
\text { studies }\end{array}$ & \multirow{2}{*}{$\begin{array}{c}\text { No. of } \\
\text { patients }\end{array}$} & Risk ratio & & \multicolumn{2}{|l}{ Model } & \multicolumn{2}{|c}{ Heterogeneity } \\
\cline { 7 - 8 } & & & OR (95\%Cl) & PoR & & $\mathrm{I}^{2}(\%)$ & $\mathrm{P}$ \\
\hline Overall & 7 & 770 & $1.53(1.06-2.21)$ & 0.02 & Random model & 65 & 0.01 \\
\hline Ethnicity & & & & & & & \\
\hline Asian & 3 & 272 & $2.94(1.71-5.05)$ & 0.02 & Random model & 55 & 0.03 \\
\hline Caucasian & 4 & 498 & $1.35(0.93-1.94)$ & 0.11 & Fixed model & 36 & 0.2 \\
\hline Follow time (months) & & & & & & & \\
\hline$\geq 100$ & 4 & 258 & $1.12(0.82-1.53)$ & 0.48 & Fixed model & 0 & 0.79 \\
\hline$<100$ & 3 & 292 & $3.35(1.65-6.84)$ & 0.0009 & Random model & 52 & 0.13 \\
\hline Cut-off & & & & & & & \\
\hline Mean & 3 & 230 & $1.75(1.16-2.62)$ & 0.007 & Random model & 57 & 0.06 \\
\hline Median & 3 & 432 & $1.39(0.87-2.21)$ & 0.17 & Fixed model & 44 & 0.17 \\
\hline ROC curve & 1 & 108 & $5.79(1.61-20.79)$ & 0.007 & - & - & - \\
\hline Sample size & & & & & & & \\
\hline$\geq 100$ & 4 & 432 & $1.59(1.16-2.18)$ & 0.004 & Fixed model & 44 & 0.09 \\
\hline$<100$ & 3 & 338 & $1.91(0.69-5.28)$ & 0.21 & Random model & 52 & 0.04 \\
\hline
\end{tabular}

\section{Sensitivity analysis}

A sensitivity analysis, in which one study was deleted at a time, was performed to gauge result stability. The results are shown in Figure $3 \mathrm{~A}$ and $\mathrm{B}$. Both of the corresponding pooled RRs of OS and DFS did not significantly change, which suggests the robustness of the results.

A

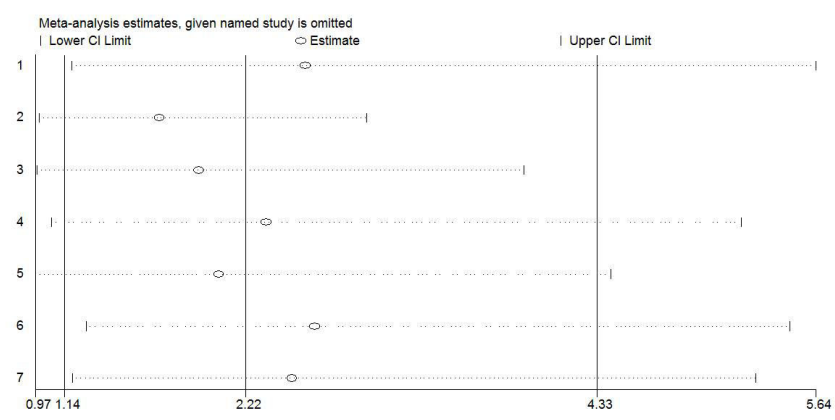

B

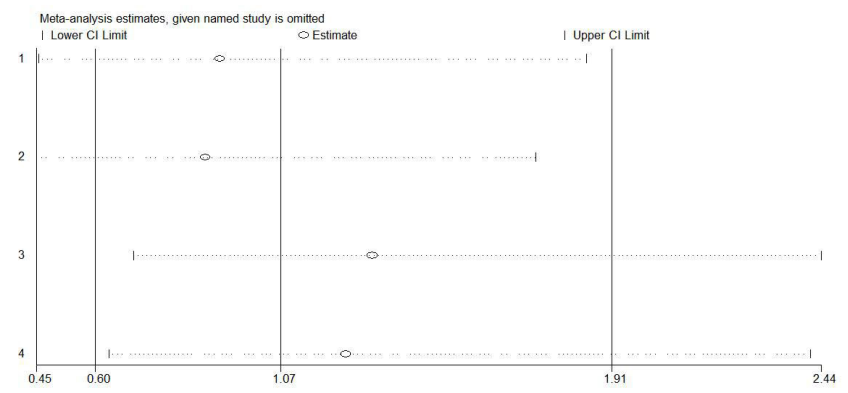

Figure 3. Sensitivity analysis of all the studies assessing disease-free survival (A) and overall survival (B). 


\section{Publication bias analysis}

The funnel plots presented no evidence of publication bias in the studies of either outcome (Figure 4A and B). No evidence for significant publication bias was found in OS (Egger test, $\mathrm{P}=$ 0.174 ) and DFS (Egger test, $P=0.293$ ) studies.
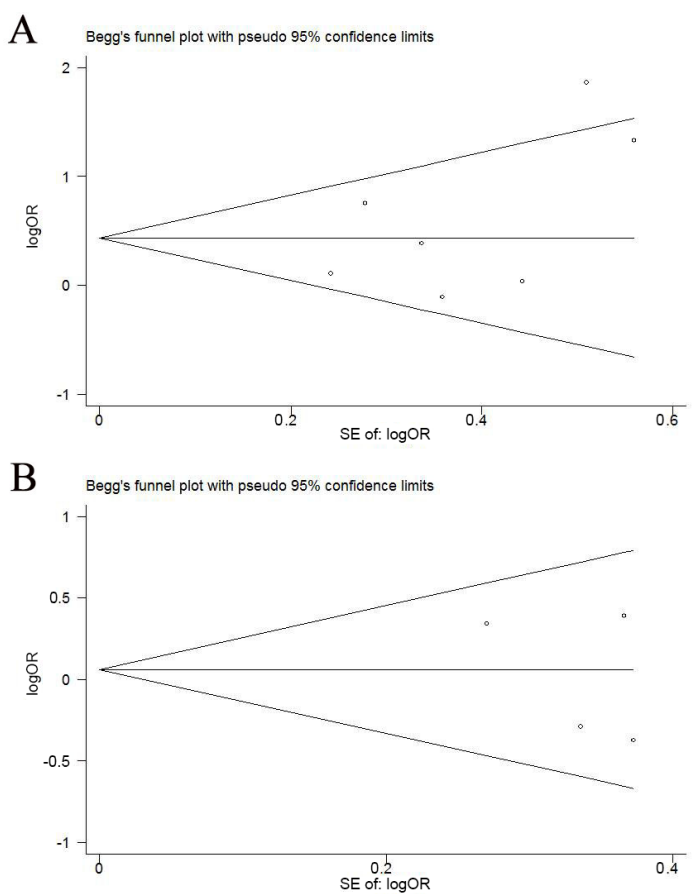

Figure 4. Begg's funnel plot estimated the publication bias of the included literature for disease-free survival (A) and overall survival (B).

\section{DISCUSSION}

Metastasis is the major cause of mortality in cancer and affects the clinical progression of cancer patients (Chiang and Massagué, 2008). MicroRNA-10b was identified as an miRNA highly expressed in metastatic breast cancer cell lines, able to generate metastases when growing as primary mammary tumor in mice (Ma et al., 2007). Several recent studies show that increased miR-10b levels have been associated with bone (Zhao et al., 2012) and brain metastases (Chiang and Massagué, 2008) as well as spread to the lymph nodes (Chen et al., 2013) in patients with breast cancer. Whether miR-10b is a prognostic marker in patients with breast cancer has been studied extensively, but the conclusions are inconsistent. To the best of our knowledge, this is the first meta-analysis of published studies to evaluate the association between miR-10b expression and prognosis in breast cancer. Heterogeneity analysis and sensitivity analysis were also critically performed to ensure the epidemiological credibility of this meta-analysis.

This meta-analysis showed that estimates of the significance of miR-10b overexpression vary substantially between studies. However, we found that high miR-10b expression was 
associated with poor DFS in patients with breast cancer, although not significant for OS. The results increase the likelihood that high miR-10b expression is an independent risk factor for breast cancer. Furthermore, miR-10b may be a therapeutic target for breast cancer because of the high expression rate of miR-10b in breast cancer lesions.

Of late years, mounting evidence by meta-analysis also shows that miR-10b overexpression is associated with aggressive clinicopathological parameters and unfavorable prognosis in several human malignancies. Wang et al. (2013) found that miR-10b was highly expressed in gastric cancer and correlated with size of tumor, Lauren classification, depth of invasion, lymph node and distant metastasis, TNM stage, and prognosis. Nakayama et al. (2013) reported that miR$10 \mathrm{~b}$ overexpression may induce an increase of pro-metastatic gene products and contribute to the acquisition of metastatic phenotypes in epithelial ovarian cancer cells. Jikuzono et al. (2013) demonstrated that the expression levels of miR-10b were shown to be upregulated in widely invasive follicular thyroid carcinoma that has distant metastasis and worse prognosis. Nakata et al. (2011) showed that miR-10b expression prompted the invasive of pancreatic cancer cells and was correlated with the prognosis of patients with pancreatic cancer. Moreover, miR-10b overexpression might be an unfavorable prognostic and a chemoradiation resistance predictive factor for colorectal cancer (Nishida et al., 2012). These findings further highlight that miR-10b may play an important role in cancer prognosis.

However, this meta-analysis has some limitations. First, the number of included studies, as well as the included breast cancer patients in each study, is relatively small. Thus, these factors might have reduced the power and accuracy of subcategory analysis. Second, the OS and DFS outcomes were based on individual unadjusted RRs. Thus, a more precise assessment should be adjusted using other prognostic factors. Third, no clear guidelines are available as regards the methods used for the evaluation of the levels of miR-10b in breast cancer patients. Such evaluation differs among all the studies. In the assessment of biomarkers, the use of a standard threshold has great importance. Although RT-PCR was the only method to analyze miR-10b expression in breast cancer specimens, differences in the cut-off values for the miR-10b overexpression may have contributed to the observed heterogeneity. Subgroup analyses showed that high miR $10 \mathrm{~b}$ expression is associated with poor DFS only when miR-10b expression level was stratified by mean value. Thus, standardized methods and cut points that classify miR-10b overexpression levels are urgently needed.

In conclusion, despite the limitations of this meta-analysis, our study suggests that miR$10 \mathrm{~b}$ expression is significantly associated with worse prognosis in terms of shorter DFS in patients with breast cancer. Hopefully this analysis will stimulate further research with rigid criteria and large study populations to resolve any remaining controversy of the role of miR-10b overexpression for the prognosis of breast cancer patients.

\section{Conflicts of interest}

The authors declare no conflict of interest.

\section{REFERENCES}

Begg CB and Mazumdar M (1994). Operating characteristics of a rank correlation test for publication bias. Biometrics 50: 10881101.http://dx.doi.org/10.2307/2533446

Chang C-H, Fan T-C, Yu J-C, Liao G-S, et al. (2014). The prognostic significance of RUNX2 and miR-10a/10b and their interrelationship in breast cancer. J. Transl. Med. 12: 257.http://dx.doi.org/10.1186/s12967-014-0257-3 
Chen W, Cai F, Zhang B, Barekati Z, et al. (2013). The level of circulating miRNA-10b and miRNA-373 in detecting lymph node metastasis of breast cancer: potential biomarkers. Tumour Biol. 34: 455-462. http://dx.doi.org/10.1007/s13277-012-0570-5

Chiang AC and Massagué J (2008). Molecular basis of metastasis. N. Engl. J. Med. 359: 2814-2823. http://dx.doi.org/10.1056/ NEJMra0805239

Egger M, Davey Smith G, Schneider M and Minder C (1997). Bias in meta-analysis detected by a simple, graphical test. BMJ 315: 629-634. http://dx.doi.org/10.1136/bmi.315.7109.629

Foley NH, Bray I, Watters KM, Das S, et al. (2011). MicroRNAs 10a and 10b are potent inducers of neuroblastoma cell differentiation through targeting of nuclear receptor corepressor 2. Cell Death Differ. 18: 1089-1098. http://dx.doi. org/10.1038/cdd.2010.172

Gee HE, Camps C, Buffa FM, Colella S, et al. (2008). MicroRNA-10b and breast cancer metastasis. Nature 455: E8-E9, author reply E9. http://dx.doi.org/10.1038/nature07362

Harbeck N (2007). Advances in breast cancer treatment. Eur. Oncol. Dis. 54-55

Higgins JP, Thompson SG, Deeks JJ and Altman DG (2003). Measuring inconsistency in meta-analyses. BMJ 327: 557-560. http://dx.doi.org/10.1136/bmj.327.7414.557

Jikuzono T, Kawamoto M, Yoshitake H, Kikuchi K, et al. (2013). The miR-221/222 cluster, miR-10b and miR-92a are highly upregulated in metastatic minimally invasive follicular thyroid carcinoma. Int. J. Oncol. 42: 1858-1868.

Karlsson E, Pérez-Tenorio G, Amin R, Bostner J, et al. (2013). The mTOR effectors 4EBP1 and S6K2 are frequently coexpressed, and associated with a poor prognosis and endocrine resistance in breast cancer: a retrospective study including patients from the randomised Stockholm tamoxifen trials. Breast Cancer Res. 15: R96. http://dx.doi.org/10.1186/bcr3557

Liang C, Tan HX, Liu WZ and Wang XF (2011). The expression of microRNA-10b in triple-negative breast cancer tissue. Zhejiang Clin. Med 13: 726-728.

Ma L, Teruya-Feldstein $\mathrm{J}$ and Weinberg RA (2007). Tumour invasion and metastasis initiated by microRNA-10b in breast cancer. Nature 449: 682-688. http://dx.doi.org/10.1038/nature06174

Markou A, Yousef GM, Stathopoulos E, Georgoulias V, et al. (2014). Prognostic significance of metastasis-related microRNAs in early breast cancer patients with a long follow-up. Clin. Chem. 60: 197-205. http://dx.doi.org/10.1373/ clinchem.2013.210542

Nakata K, Ohuchida K, Mizumoto K, Kayashima T, et al. (2011). MicroRNA-10b is overexpressed in pancreatic cancer, promotes its invasiveness, and correlates with a poor prognosis. Surgery 150: 916-922. http://dx.doi.org/10.1016/j. surg.2011.06.017

Nakayama I, Shibazaki M, Yashima-Abo A, Miura F, et al. (2013). Loss of HOXD10 expression induced by upregulation of miR$10 \mathrm{~b}$ accelerates the migration and invasion activities of ovarian cancer cells. Int. J. Oncol. 43: 63-71.

Nishida N, Yamashita S, Mimori K, Sudo T, et al. (2012). MicroRNA-10b is a prognostic indicator in colorectal cancer and confers resistance to the chemotherapeutic agent 5-fluorouracil in colorectal cancer cells. Ann. Surg. Oncol. 19: 30653071. http://dx.doi.org/10.1245/s10434-012-2246-1

Parrella P, Barbano R, Pasculli B, Fontana A, et al. (2014). Evaluation of microRNA-10b prognostic significance in a prospective cohort of breast cancer patients. Mol. Cancer 13: 142. http://dx.doi.org/10.1186/1476-4598-13-142

Radojicic J, Zaravinos A, Vrekoussis T, Kafousi M, et al. (2011). MicroRNA expression analysis in triple-negative (ER, PR and Her2/neu) breast cancer. Cell Cycle 10: 507-517. http://dx.doi.org/10.4161/cc.10.3.14754

Sasayama T, Nishihara M, Kondoh T, Hosoda K, et al. (2009). MicroRNA-10b is overexpressed in malignant glioma and associated with tumor invasive factors, uPAR and RhoC. Int. J. Cancer 125: 1407-1413. http://dx.doi.org/10.1002/ ijc. 24522

Song CG, Fu FM, Wu XY, Han ZH, et al. (2012). Correlation of miR-10b with invasiveness and prognosis of breast cancer. Chin. Oncol. J. 22: 31-34.

Wang Y-Y, Ye Z-Y, Zhao Z-S, Li L, et al. (2013). Clinicopathologic significance of miR-10b expression in gastric carcinoma. Hum. Pathol. 44: 1278-1285. http://dx.doi.org/10.1016/j.humpath.2012.10.014

Zhao FL, Hu GD, Wang XF, Zhang XH, et al. (2012). Serum overexpression of microRNA-10b in patients with bone metastatic primary breast cancer. J. Int. Med. Res. 40: 859-866. http://dx.doi.org/10.1177/147323001204000304 